\title{
Dose-Efficient Cryo-STEM Imaging of Whole Cells Using the Electron Microscope Pixel Array Detector
}

\author{
Katherine A. Spoth ${ }^{1}$, Kayla X. Nguyen ${ }^{1}$, David A. Muller ${ }^{1,2}$, and Lena F. Kourkoutis ${ }^{1,2}$ \\ 1. School of Applied and Engineering Physics, Cornell University, Ithaca, NY, USA. \\ 2. Kavli Institute at Cornell for Nanoscale Science, Ithaca, NY, USA.
}

For specimens thicker than the inelastic mean free path $(\sim 300 \mathrm{~nm}$ at $300 \mathrm{kV})$, such as whole cells, STEM imaging has shown better performance than TEM because it lacks chromatic blurring caused by postspecimen imaging optics in the TEM. This advantage is particularly useful for electron tomography of both embedded [1] and cryo-preserved cells [2]. However, typical STEM detector geometries-either an annular detector collecting electrons scattered to high angles or an on-axis bright field detector collecting the forward-scattered beam-make use of only a small fraction of the incident electron dose.

Here, we demonstrate the use of an electron microscope pixel array detector (EMPAD) for high-doseefficiency STEM imaging of fully hydrated, vitrified $E$. coli whole cells [3]. The detector's high dynamic range of 1,000,000:1 allows collection of the full convergent beam electron diffraction (CBED) pattern at each probe position with single-electron sensitivity. This has important consequences for low-dose imaging: the entire dose incident on the specimen is collected by the detector, resolved by both angle and position simultaneously, allowing flexibility in controlling the signal contributing to the final image as well as novel imaging modes.

STEM imaging with the EMPAD is illustrated in Figure 1. Images were taken at $300 \mathrm{kV}$ with the probe convergence angle set to $2 \mathrm{mrad}$ to provide large depth of field for imaging cells approximately $500-600$ $\mathrm{nm}$ thick. The total dose on the specimen was $7.5 \mathrm{e}^{-} / \AA^{2}$. The CBED pattern collected at one scan position is shown along with overlays of the geometries used to calculate the annular dark field (ADF) and bright field (BF) STEM signal for each of the 256x256 scan pixels. The conventional BF-STEM image uses only $6 \%$ of the incident dose. Cellular features are visible, but the SNR is low and the resolution is limited due to the large pixel size. Considerable improvements are obtained by forming a BF image using a greater number of pixels from the bright field disk. Each detector pixel acts as a separate BF detector forming its own image. Due to reciprocity, using pixels off the optical axis is equivalent to tilting the beam for TEM illumination which causes a shift in the resulting images [4]. To prevent blurring of the final BF image and allow for sub-pixel sampling, individual images from each detector pixel are aligned by crosscorrelation, resized, and summed. With this tilt-corrected BF-STEM (i.e. tcBF-STEM) technique, we collect 5 times more electrons than in the conventional BF detector geometry while retaining coherency.

The tcBF-STEM technique is compared to TEM and zero-loss EFTEM of the same specimen area with the same dose on the specimen $\left(7.5 \mathrm{e}^{-} / \AA^{2}\right)$ in Figure 2. TEM images are defocused by $-7.5 \mu \mathrm{m}$ while the STEM image showing similar contrast was acquired with only $-1 \mu \mathrm{m}$ defocus. We clearly resolve the membrane bilayer in the STEM image (Figure 2, insets), most likely due to the lower defocus used in this mode. In the thicker part of the cell, the TEM image shows low contrast due to inelastic scattering that results in phase incoherence and chromatic blurring. With these electrons filtered out, the EFTEM image retains contrast in the thicker region; however, SNR is significantly reduced due to loss of electrons from energy filtering. The tcBF-STEM image shows the most detail at the center of the cell, suggesting this technique will be well suited for tomographic imaging of thick specimens [5]. 
[1] A. A. Sousa and R. D. Leapman, Ultramicroscopy 123 (2012), p. 38-49.

[2] S. G. Wolf, L. Houben, and M. Elbaum, Nature Methods 11 (2014), p. 423-428.

[3] M. W. Tate, et al, Microscopy and Microanalysis 22 (2016), p. 237-249.

[4] A. J. Koster and A. F. de Jong, Ultramicroscopy 38 (1991), p. 235-240.

[5] Work supported by the Packard Foundation and NSF awards (DMR-1429155 and DMR-1120296).

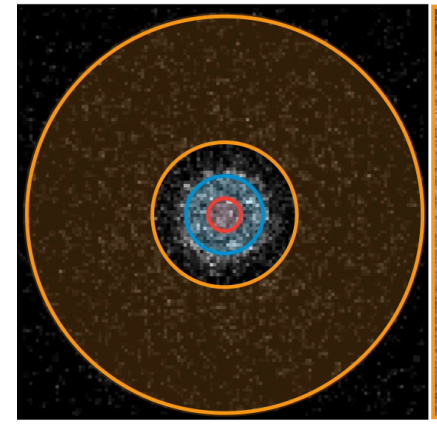

Annular Dark Field

$37 \%$ of incident dose

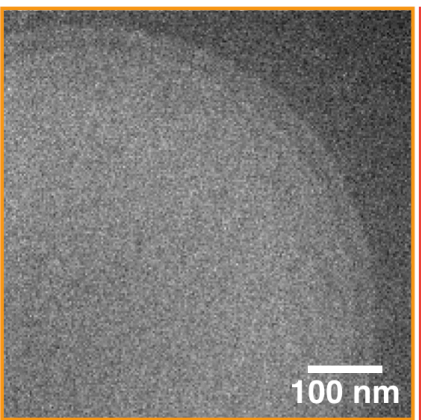

\section{Bright Field}

$6 \%$ of incident dose

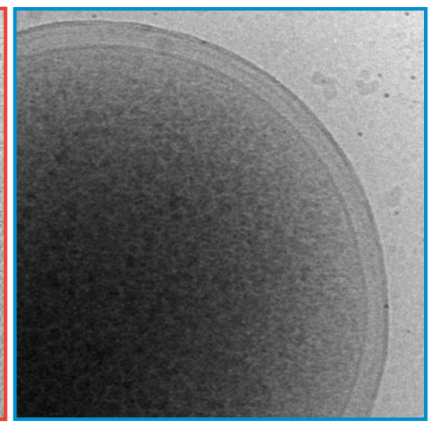

Tilt-Corrected Bright Field $32 \%$ of incident dose

Figure 1. CBED pattern collected on the EMPAD at one scan position (left). Annular dark field (ADF) and bright field (BF) images formed with conventional STEM detector geometries are shown, with ADF corresponding to the outermost shaded annulus and BF to the inner circle. The tcBF-STEM image is the sum of cross-correlated images formed from each pixel in the central disk of the CBED pattern, using 5 times more of the incident dose than the conventional BF-STEM image.
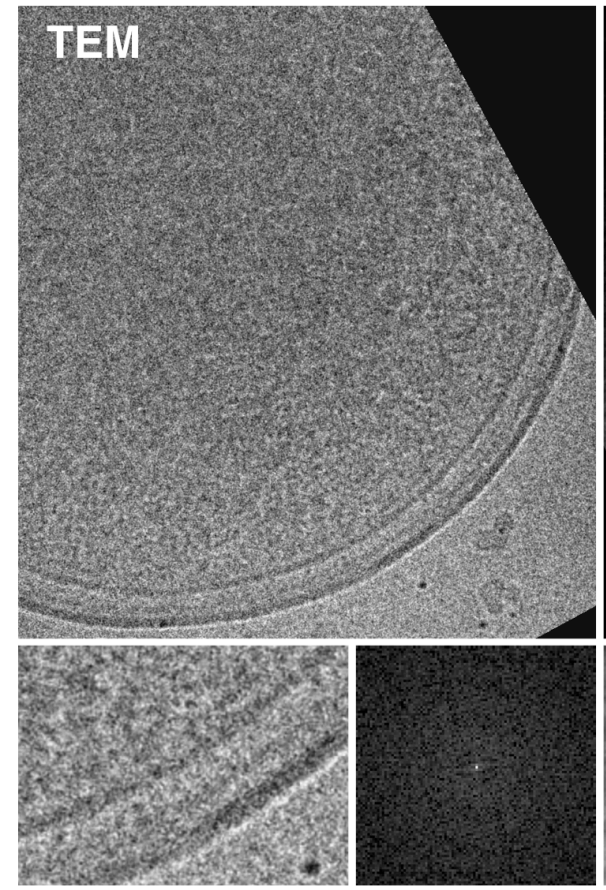

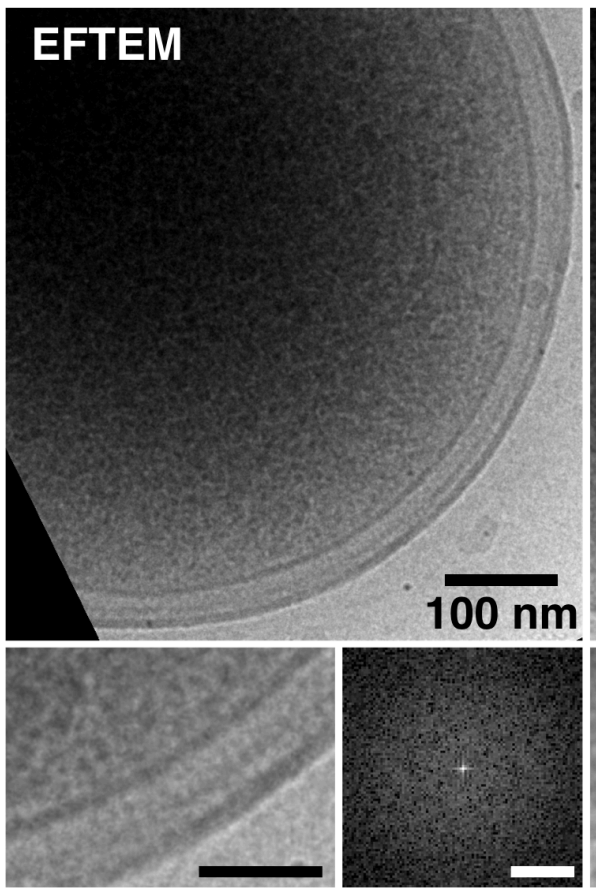

tcBF-STEM

Figure 2. Comparison of STEM imaging using the EMPAD with conventional TEM and zero-loss EFTEM for the same sample area at the same dose $\left(7.5 \mathrm{e}^{-} / \AA^{2}\right)$. TEM shows low contrast in thick regions while the EFTEM image is dominated by the thickness gradient towards the center. tcBF-STEM shows distinguishable ribosomes even in thick regions of the cell. The membrane bilayer is clearly resolved in the STEM image. Comparing FFTs from thick regions of the cell for each imaging mode shows enhanced information transfer for STEM in this region. Scale bars: inset image $50 \mathrm{~nm}$; FFT $0.1 \mathrm{~nm}^{-1}$. 